\title{
Chiral Properties of Strong Interactions in a Magnetic Background
}

\section{D’Elia}

Dipartimento di Fisica dell'Università di Genova, I-16146 Genova, Italy and INFN, Sezione di Genova, I-16146 Genova, Italy

E-mail: massimo.delia@ge.infn.it

\section{F. Negro*}

Dipartimento di Fisica dell'Università di Genova, I-16146 Genova, Italy and INFN, Sezione di Genova, I-16146 Genova, Italy

E-mail: fnegro@ge.infn.it

We discuss the chiral properties of $N_{f}=2 \mathrm{QCD}$ in presence of a strong magnetic field, as they emerge from lattice numerical simulations in the low temperature regime. We focus on magnetic catalysis, i.e. the increase of chiral symmetry breaking induced by the background field. We show that a substantial contribution to magnetic catalysis comes from the modified distribution of non-Abelian gauge fields, induced by the magnetic field via dynamical quark loop effects.

XXIX International Symposium on Lattice Field Theory

July 10-16, 2011

Squaw Valley, Lake Tahoe, California

\footnotetext{
${ }^{*}$ Speaker.
} 


\section{Introduction}

The study of strong interactions in presence of very intense magnetic background fields has revealed to be of great interest, going from the study of cosmological transitions, to the phenomenology of heavy ion collisions and of compact astrophysical objects such as magnetars. There has been an increasing number of related lattice studies, mostly dedicated to the investigation of the fate of the deconfinement transition in presence of a background chromo-magnetic [1,2] or magnetic $[3,4]$ background field, and to the study of the chiral magnetic effect [5].

In the present study we address the issue of magnetic catalysis, i.e. the enhancement of chiral symmetry breaking induced by the magnetic field, a phenomenon predicted from different low energy models and approximations of QCD [6, 7, 8, 9, 10, 11, 12]. Previous lattice studies have considered the effect of the magnetic field on quenched configurations [13, 14]. Instead we consider full QCD with two dynamical flavors carrying different electric charges, corresponding respectively to the $u$ and $d$ quark charges, and coupled to a background constant and uniform magnetic field.

We have considered a symmetric lattice, $L_{x}=L_{y}=L_{z}=N_{t}=16$, with periodic boundary conditions in space and thermal conditions in the time, a bare quark mass $a m=0.01335$ and an inverse gauge coupling $\beta=5.30$. According to scale estimates reported in Ref. [3], that corresponds to a lattice spacing $a \simeq 0.3 \mathrm{fm}$, a (Goldstone) pion mass $m_{\pi} \simeq 200 \mathrm{MeV}$ and a temperature $T=\left(N_{t} a\right)^{-1} \simeq 40 \mathrm{MeV}$. We have made use of an RHMC algorithm to simulate rooted staggered fermions. Numerical simulations have been performed on the apeNEXT facilities in Rome.

We have explored different values of $|e| B$, which on a torus is quantized according to

$$
|e| B=6 \pi b / l_{x} l_{y}=6 \pi b a^{-2} / L_{x} L_{y}
$$

where $b$ is an integer, i.e. it can be changed by integer multiples $6 \pi a^{-2} / L_{x} L_{y} \simeq(180 \mathrm{MeV})^{2}$. The presence of an ultra-violet (UV) cutoff imposes also an upper limit on the possible values of $B$, since we cannot distinguish magnetic fields giving the same phase factor, modulo $2 \pi$, to a particle moving around the minimum closed path on the lattice, i.e. the plaquette: all physical quantities are periodic in $q B$ with a period $2 \pi / a^{2}$. We can therefore define a sort of "first Brillouin zone" $-\pi / a^{2}<q B<\pi / a^{2}$, i.e. $-L_{x} L_{y} / 2<b<L_{x} L_{y} / 2$. Symmetry under $b \rightarrow-b$ further reduces the range of interesting values of $b$. One expects the periodicity to induce saturation effects which could affect lattice results.

The purpose of our investigation is to study the dependence of the chiral condensate on the magnetic field and to compare it with existing predictions. Moreover we want to understand which part of magnetic catalysis is a purely tree level effect, due to the fact that quarks propagate in a modified background obtained by adding the $\mathrm{U}(1)$ field to the non-Abelian gauge configurations, and which part is due to a modification of the non-Abelian fields themselves, induced by the loop effects of dynamical quarks coupled to the magnetic background. Both effects can in principle modify the spectrum of the Dirac operator, leading to an increase of the chiral condensate.

In presence of a non-zero $B$ we can define two different condensates

$$
\left.\Sigma_{u / d}(B) \equiv \frac{\partial \log Z}{\partial m_{u / d}}\right|_{m_{u / d}=m}=\int \mathscr{D} U \mathscr{P}[m, U, B] \operatorname{Tr}\left(M^{-1}\left[m, B, q_{u / d}\right]\right)
$$

where $M$ is the fermion matrix, depending on $B$ and on the quark mass and charge, and the functional integral measure is $\mathscr{P}[m, U, B] \propto \operatorname{det} M^{\frac{1}{4}}\left[m, B, q_{u}\right] \operatorname{det} M^{\frac{1}{4}}\left[m, B, q_{d}\right] e^{-S_{G}}$. 
In the following we shall make use of the relative increment of $\Sigma$, which is defined as:

$$
r_{u / d}(B) \equiv \frac{\Sigma_{u / d}(B)}{\Sigma(0)}-1=\frac{\Sigma_{u / d}(B)-\Sigma(0)}{\Sigma(0)}
$$

and for which most renormalizations affecting the definition of $\Sigma$ cancels out. The magnetic field, contrary to the quark mass, does not break explicitly all chiral symmetry generators $\left(\gamma_{5} \tau_{3}\right.$ is preserved); assuming that the additive mass dependent renormalization of $\Sigma$ has a negligible dependence on $B$, at least for $B$ well below the UV cutoff, such renormalization will cancel out in the numerator of Eq. (1.3). A residual additive renormalization remains in the denominator, leading to an incorrect overall normalization of $r(B)$, which one can estimate, in our case, to be $O(10 \%)$ [15].

We shall make use of flavor averaged quantities as well, $\Sigma(B)=\left(\Sigma_{u}(B)+\Sigma_{d}(B)\right) / 2$ and $r(B)=\Sigma(B) / \Sigma(0)-1=\left(r_{u}(B)+r_{d}(B)\right) / 2$. Both $\Sigma_{u}(B)$ and $\Sigma_{d}(B)$ are, by charge conjugation symmetry, even functions of $B$; moreover they are periodic in $B$, or equivalently in $b$ with a period $L_{x} L_{y}$.

We define also the "valence" contribution, deriving purely from the change in the observable:

$$
\Sigma_{u / d}^{v a l}(B) \equiv \int \mathscr{D} U \mathscr{P}[m, U, 0] \operatorname{Tr}\left(M^{-1}\left[m, B, q_{u / d}\right]\right),
$$

the "dynamical" one, deriving purely from the change in the measure:

$$
\Sigma_{u / d}^{d y n}(B) \equiv \int \mathscr{D} U \mathscr{P}[m, U, B] \operatorname{Tr}\left(M^{-1}\left[m, 0, q_{u / d}\right]\right) .
$$

and from them the corresponding quantities $\Sigma^{\mathrm{val} / \mathrm{dyn}}, r_{u / d}^{\mathrm{val} / \mathrm{dyn}}, r^{\mathrm{val} / \mathrm{dyn}}$.

On general grounds we may expect that, in the limit of small fields, $B$ acts as a perturbation for both the measure term $\mathscr{P}[m, U, B]$ and the observable $\operatorname{Tr}\left(M^{-1}\left[m, B, q_{u / d}\right]\right)$ in Eq. (1.2). In this case one can show [15] that

$$
\frac{\Sigma_{u / d}(B)}{\Sigma(0)}-1=r_{u / d}^{v a l}(B)+r_{u / d}^{d y n}(B)+O\left(B^{4}\right) .
$$

Therefore, at least in the limit of small fields, the separation of magnetic catalysis in a valence part and in a dynamical part is a well defined concept.

A full account of our results and more details about our implementation of QCD in presence of a background magnetic field can be found in Ref. [15].

\section{Results}

In Fig. 1 we report results for the normalized condensates $\Sigma_{u / d}(B) / \Sigma(B)$ (i.e. $1+r_{u / d}(B)$ ) over the whole range of possible independent values of $B$, i.e. for $b /\left(L_{x} L_{y}\right)$ ranging from 0 to 1 . Saturation effects, which are present for large values of $B$, are clearly visible from Fig. 1: one should keep $b /\left(L_{x} L_{y}\right)$ well below 0.1 in order that such effects stay negligible.

Notice that the $u$ quark condensate shows an approximate periodicity in $B$ which is halved with respect to the $d$ quark. That comes from the fact that $\left|q_{u}\right|=2\left|q_{d}\right|$ and is only approximate since instead the measure term has the usual periodicity. 


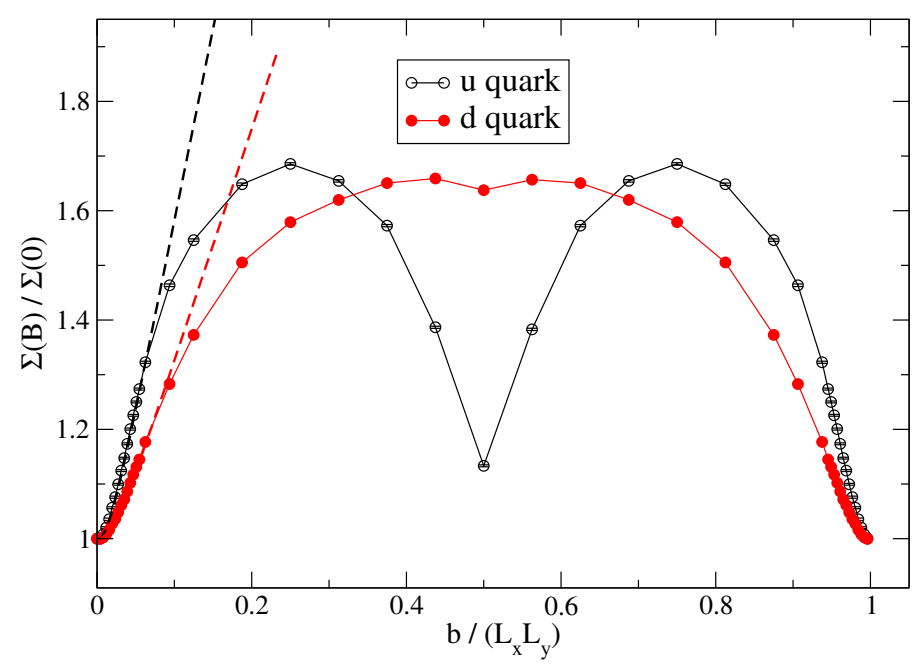

Figure 1: Normalized $u$ and $d$ quark condensates as a function of the magnetic field for the whole range of independent possible values of $B$. Data for $b /\left(L_{x} L_{y}\right)>0.6$ have been obtained by enforcing the expected symmetry under $b /\left(L_{x} L_{y}\right) \rightarrow 1-b /\left(L_{x} L_{y}\right)$. We also report two curves corresponding to best fits in the small field region, to better show the presence of saturation effects.

In Fig. 2 we report the functions $r(B), r^{v a l}(B), r^{d y n}(B)$ (see Eqs. (1.4) and (1.5)) and their sum, in order to appreciate the amount of magnetic catalysis caused by the modified distribution of gauge fields induced by the coupling of dynamical quarks to the magnetic field. We have limited our analysis to $b \leq 16$, for which saturation effects do not play a significant role.

The first thing that we notice is that the dynamical and valence contributions are roughly additive: their sum gives back to full signal, in the range of fields shown in the figure. The additivity, which is expected in the limit of small fields, is verified within errors for $b \leq 8\left(|e| B \leq(500 \mathrm{MeV})^{2}\right)$, while small deviations appear beyond. Once clarified that it is sensible, in the explored range of fields, to divide magnetic catalysis into a valence and a dynamical contribution, from Fig. 2 we learn that the dynamical one is roughly $40 \%$ of the total signal, at least for the discretization and quark mass spectrum adopted in our investigation; hence quenching effects, which are typically of the order of $20 \%$, may be larger in the case of magnetic catalysis.

One of the purposes of our investigation is to compare our results with various analytic predictions. One of the first was based on the analysis of the Nambu - Jona-Lasino model [7] and predicted a quadratic increase of the condensate as a function of the magnetic field, i.e. $r(B) \propto B^{2}$.

The first prediction based on chiral perturbation theory has been proposed in Ref. [9], leads to $r(B)=\log (2)|e| B /\left(16 \pi^{2} F_{\pi}^{2}\right)$ and is valid only in the chiral limit, i.e. $m_{\pi}=0$, and for $|e| B \ll \Lambda_{\mathrm{QCD}}^{2}$. The authors of Ref. [11] have gone beyond the limitation $m_{\pi}=0$, presenting a $\chi \mathrm{PT}$ computation which is valid for generic values of $m_{\pi}^{2} /(|e| B)$, even if still for $|e| B \ll \Lambda_{\mathrm{QCD}}^{2}$; the prediction is:

$$
r(B)=\frac{\log (2) e B}{16 \pi^{2} F_{\pi}^{2}} I_{H}\left(\frac{m_{\pi}^{2}}{|e| B}\right) ; I_{H}(y)=\frac{1}{\log 2}\left(\log (2 \pi)+y \log \left(\frac{y}{2}\right)-2 \log \Gamma\left(\frac{1+y}{2}\right)\right) .
$$

A range of other possible behaviors, including a power law dependence, has been predicted by means of the AdS/CFT correspondence. 


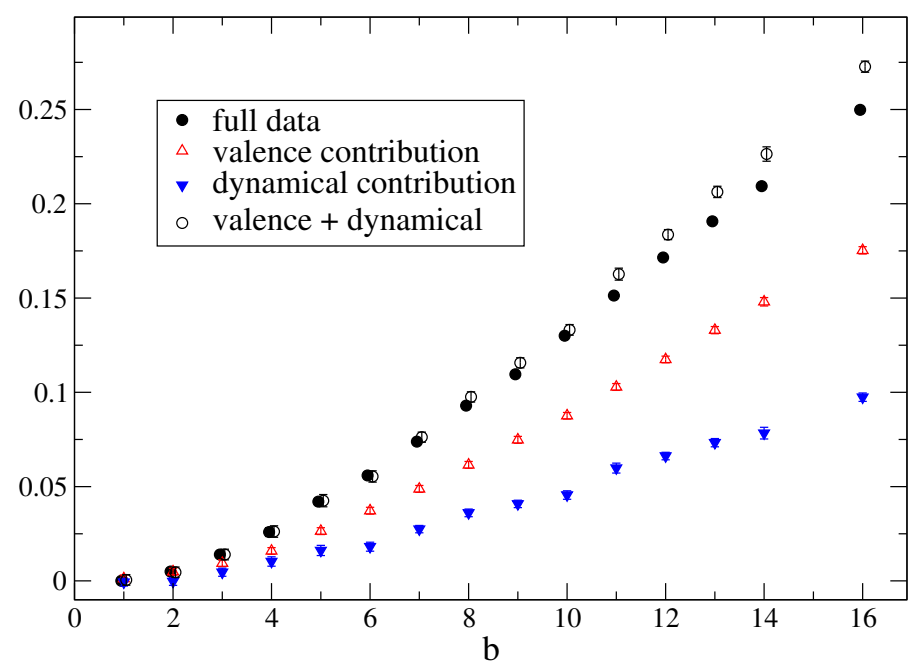

Figure 2: Relative increment of the average of the $u$ and $d$ quark condensates as a function of the magnetic field. We report separately $r(B), r^{v a l}(B), r^{d y n}(B)$ and $r^{v a l}(B)+r^{d y n}(B)$.

Existing lattice determinations have reported a linear behaviour for $\mathrm{SU}(2)$ pure gauge theory [13] and a power law behavior $r(B) \propto B^{v}$ (with $v \sim 1$.6) for the $\mathrm{SU}(3)$ pure gauge theory [14].

In Fig. 3 we report the relative increment of the $u$ and $d$ condensates and of their average in a restricted region for which we expect that saturation effects are not important. It is apparent by eye that a linear behavior badly fits with our data, giving unacceptable values for the $\chi^{2} /$ d.o.f. test (e.g. $\chi^{2} /$ d.o.f. $\simeq 190 / 6$ for $1 \leq b \leq 7$ ). This is expected, since in our case $m_{\pi} \neq 0$.

Next we have tried to check if a quadratic behavior $r(B)=\left(|e| B / \Lambda_{B}^{2}\right)^{2}$ fits better, at least for small enough fields. For $b<8$, i.e. $|e| B<(500 \mathrm{MeV})^{2}$, the quadratic fit looks good and stable, with $\Lambda_{B} \sim 900 \mathrm{MeV}$.

We have then tried to fit our data with the prediction of Ref. [11], as reported in Eq. (2.1). We have obtained reasonable fits only if both $m_{\pi}$ and $F_{\pi}$ are treated as independent free parameters. Typical values of the fit parameters are $m_{\pi} \sim 300-400 \mathrm{MeV}$ and $F_{\pi} \sim 60-70 \mathrm{MeV}$. The fitted pion mass is somewhat larger than the value obtained, with the same discretization settings, by measuring meson correlators, i.e. $m_{\pi} \sim 200 \mathrm{MeV}$ [3]: one possible explanation is in the explicit flavour symmetry breaking induced by the staggered discretization: the three pions are not degenerate in mass and what is determined by meson correlator measurements is just the lowest pion mass. Regarding $F_{\pi}$, since it enters Eq. (2.1) only in the prefactor, its value is surely affected by the systematic uncertainty in the overall normalization factor for $r(B)$, which is of the order of $10 \%$. One should also take into account that the $\chi$ PT prediction of Ref. [11] has been obtained in the low energy limit $|e| B \ll \Lambda_{\mathrm{QCD}}^{2}$, a condition which is violated in our explored range of fields.

We have verified that other two-parameter functions, which allow to fix independently the curvature at $B=0$ and the asymptotic linear behavior for larger fields (like Eq. (2.1) when $F_{\pi}$ and $m_{\pi}$ are treated as independent parameters) work equally well. For instance the function

$$
r(b)=c_{0} b \operatorname{atan}\left(c_{1} b\right)
$$

fits well in the whole range of explored fields, $b_{\min }=1$ and $b_{\max }=16$, with $c_{0}=0.0136(2)$, $c_{1}=0.140(5)$ and $\chi^{2} /$ d.o.f. $\simeq 0.77$. 


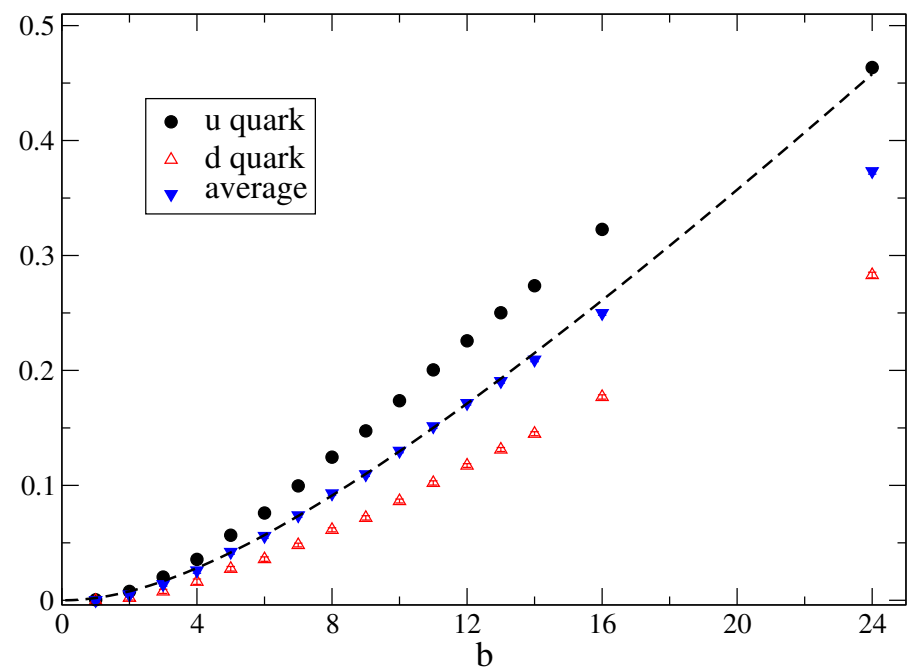

Figure 3: Relative increment of the quark condensate as a function of the magnetic field. We report separately data for the $u$ and $d$ quarks as well as for the average of the two, together with a best fit to Eq. (2.1).

To compare with the analysis performed in Ref. [14], we have also investigated if a power law behaviour $r(B)=\left(|e| B / \Lambda_{B}^{2}\right)^{v}$ can fit our data. Reasonable fits are obtained only for a range of fields including $b=8$ : values obtained for $v$ in this range are roughly compatible with $v=2$.

\section{Conclusions}

In this work we have probed $N_{f}=2$ QCD with an external uniform magnetic field. We have studied the phenomenon of magnetic catalysis, i.e. the breaking of chiral symmetry as a function of the test field, by means of numerical lattice simulations.

The main result we have shown is that, in the range $|e| B \in\left[(180 \mathrm{MeV})^{2} ;(700 \mathrm{MeV})^{2}\right]$, it is possible to divide magnetic catalysis into two contributions. The dynamical one comes from the modified distribution of non-Abelian gauge fields, induced by dynamical quark loop effects. The valence one comes from the effect of the magnetic field on the valence quarks and can be determined by measuring the condensate on gauge configurations sampled with the unmodified distribution. The first term, which is missed by quenched or partially quenched studies, accounts for about $40 \%$ of the total increase in the quark condensate.

Regarding the dependence of the condensate on the magnetic field, we have shown that a quadratic behavior, which is expected in the limit of small magnetic fields, describes well our data for $|e| B$ up to $\sim(500 \mathrm{MeV})^{2}$. The $\chi \mathrm{PT}$ prediction of Ref. [11] fits data over a wider range, but only if the pion decay constant and mass are treated as independent free parameters.

We address to further studies the possibility to improve the simulation in order to overcome the problems we had to deal with. In particular an improved lattice action formulation and a finer spacing $a$ would allow to check for lattice artifacts, to test the correct scaling to the continuum limit of our results and to explore larger values of the magnetic field. Instead larger spatial volumes would allow for a finer quantization of $|e| B$ and for a better investigation of the small field region. It would be also interesting to explore different choices of the quark mass spectrum, in order to see 
how the separation of magnetic catalysis into a dynamical and a valence contribution depends on the dynamical quark masses.

\section{References}

[1] P. Cea and L. Cosmai, JHEP 0508, 079 (2005).

[2] P. Cea, L. Cosmai and M. D’Elia, JHEP 0712, 097 (2007).

[3] M. D’Elia, S. Mukherjee, F. Sanfilippo, Phys. Rev. D82, 051501 (2010). [arXiv:1005.5365 [hep-lat]].

[4] G. Endrodi et al., these proceedings.

[5] P. V. Buividovich, M. N. Chernodub, E. V. Luschevskaya and M. I. Polikarpov, Phys. Rev. D 80, 054503 (2009); M. Abramczyk, T. Blum, G. Petropoulos and R. Zhou, arXiv:0911.1348 [hep-lat]; A. Yamamoto, Phys. Rev. Lett. 107, 031601 (2011) [arXiv:1105.0385 [hep-lat]].

[6] A. Salam and J. A. Strathdee, Nucl. Phys. B 90, 203 (1975); A. D. Linde, Phys. Lett. B 62, 435 (1976); S. Kawati, G. Konisi, H. Miyata, Phys. Rev. D28, 1537-1541 (1983).

[7] S. P. Klevansky and R. H. Lemmer, Phys. Rev. D 39, 3478 (1989).

[8] H. Suganuma and T. Tatsumi, Annals Phys. 208, 470 (1991); K. G. Klimenko, Z. Phys. C 54, 323 (1992); S. Schramm, B. Muller, A. J. Schramm, Mod. Phys. Lett. A7, 973-982 (1992);

K. G. Klimenko, B. V. Magnitsky, A. S. Vshivtsev, Nuovo Cim. A107, 439-452 (1994); V. P. Gusynin, V. A. Miransky and I. A. Shovkovy, Phys. Rev. Lett. 73, 3499 (1994) [Erratum-ibid. 76, 1005 (1996)] [arXiv:hep-ph/9405262]; V. P. Gusynin, V. A. Miransky and I. A. Shovkovy, Phys. Lett. B 349, 477 (1995).

[9] I. A. Shushpanov and A. V. Smilga, Phys. Lett. B 402, 351 (1997).

[10] A. Y. .Babansky, E. V. Gorbar, G. V. Shchepanyuk, Phys. Lett. B419, 272-278 (1998); D. Ebert, K. G. Klimenko, M. A. Vdovichenko and A. S. Vshivtsev, Phys. Rev. D 61, 025005 (2000); A. Goyal, M. Dahiya, Phys. Rev. D62, 025022 (2000) [hep-ph/9906367]; N. O. Agasian and I. A. Shushpanov, Phys. Lett. B 472, 143 (2000) [arXiv:hep-ph/9911254]; D. Ebert, V. V. Khudyakov, V. C. Zhukovsky, K. G. Klimenko, Phys. Rev. D65, 054024 (2002). [hep-ph/0106110]; D. N. Kabat, K. -M. Lee, E. J. Weinberg, Phys. Rev. D66, 014004 (2002). [hep-ph/0204120]; V. A. Miransky and I. A. Shovkovy, Phys. Rev. D 66, 045006 (2002).

[11] T. D. Cohen, D. A. McGady and E. S. Werbos, Phys. Rev. C 76, 055201 (2007).

[12] C. V. Johnson and A. Kundu, JHEP 0812, 053 (2008) [arXiv:0803.0038 [hep-th]]; E. Rojas, A. Ayala, A. Bashir, A. Raya, Phys. Rev. D77, 093004 (2008). [arXiv:0803.4173 [hep-ph]]; O. Bergman, G. Lifschytz and M. Lippert, Phys. Rev. D 79, 105024 (2009) [arXiv:0806.0366 [hep-th]]; A. V. Zayakin, JHEP 0807, 116 (2008); N. Evans, T. Kalaydzhyan, K. y. Kim and I. Kirsch, JHEP 1101, 050 (2011) [arXiv:1011.2519 [hep-th]]; A. J. Mizher, E. S. Fraga, M. N. Chernodub, [arXiv:1103.0954 [hep-ph]]; S. -i. Nam, C. -W. Kao, [arXiv:1103.6057 [hep-ph]].

[13] P. V. Buividovich, M. N. Chernodub, E. V. Luschevskaya and M. I. Polikarpov, Phys. Lett. B 682, 484 (2010), Nucl. Phys. B 826, 313 (2010).

[14] V. V. Braguta, P. V. Buividovich, T. Kalaydzhyan, S. V. Kuznetsov, M. I. Polikarpov, PoS LATTICE2010, 190 (2010). [arXiv:1011.3795 [hep-lat]].

[15] M. D’Elia, F. Negro, Phys. Rev. D83, 114028 (2011). [arXiv:1103.2080 [hep-lat]]. 